\title{
ÖSTERREICHISCHE REPOSITORIEN IN OPENDOAR UND RE3DATA.ORG: ENTWICKLUNG UND STATUS VON INFRASTRUKTUREN FÜR GREEN OPEN ACCESS UND FORSCHUNGSDATEN
}

\section{von Bruno Bauer und Andreas Ferus}

Zusammenfassung: Der vorliegende Beitrag beleuchtet die Entwicklung und den Status Quo von Repositorien in Österreich. Diese haben mit dem Hochschulraumstrukturmittelprojekt e-Infrastructures Austria einen wichtigen Impuls bekommen. Während in den Jahren nach der „Berliner Erklärung über den offenen Zugang zu wissenschaftlichem Wissen" (2003) vor allem die Bereiche Publikationen und Green Open Access bearbeitet wurden, rückten in jüngster Zeit insbesondere durch die European Open Science Cloud (EOSC) auch Forschungsdaten zunehmend in den Fokus des Interesses. Dieser Aufschwung spiegelt sich auch in der laufenden Steigerung der im Directory of Open Access Repositories (OpenDOAR) und im Registry of Research Data Repositories (re3data.org) erfassten österreichischen Repositorien wider. Mittels statistischer Auswertungen wurde erhoben, welche Dokumententypen in diesen Repositorien aufgenommen werden, welche Fachgebiete sie repräsentieren, welchen Umfang sie aufweisen, welche Software eingesetzt wird, ob bereits notwendige Schnittstellen (wie z.B. OAI) vorhanden sind und welche Policies für die jeweiligen Repositorien verfolgt werden.

Schlüsselwörter: OpenDOAR; re3data.org; Repositorium; Green Open Access; Zweitveröffentlichung; Forschungsdaten; Österreich; Entwicklung; Übersicht; Statistik

\section{AUSTRIAN REPOSITORIES IN OPENDOAR AND RE3DATA.ORG: THE DEVELOPMENT OF INFRASTRUCTURES FOR GREEN OPEN ACCESS AND RESEARCH DATA}

Abstract: The article highlights the development and status quo of repositories in Austria, which have received an important impulse by the project e-Infrastructures Austria. While in the years following the "Berlin Declaration on Open Access to Scientific Knowledge" (2003), mainly the areas of publications and Green Open Access were dealt with, but recently research data has become increasingly popular, especially in the context of initiatives like the European Open Science Cloud (EOSC). This upturn is also reflected in the ongoing increase in the number of Austrian repositories listed in the Directory of Open Access Repositories (OpenDOAR) and the Registry of Research Data Repositories (re3data.org). By means of statistical evaluations it was ascertained which types of documents are included in these repositories, which 
academic disciplines they represent, the extent of their content, which software is used, whether already existing interfaces (such as OAI) are available and which policies are being followed for the respective repositories.

Keywords: OpenDOAR; re3data.org; Repository; Green Open Access, Self Archiving, Reseach Data, Austria; Development; Overview; Statistics

DOI: http://doi.org/10.31263/voebm.v71i1.2037

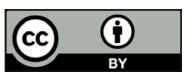

Dieses Werk ist lizenziert unter einer

Creative-Commons-Lizenz Namensnennung 4.0 International

\section{Inhalt}

\section{Einleitung}

2. Schwierige Anfangsphase für Repositorien in Österreich

3. Aufbau von Repositorien im Rahmen von e-Infrastructures Austria

4. OpenDOAR als Nachweisinstrument für Repositorien

5. Österreichische Repositorien in OpenDOAR

6. Spezifika der österreichischen Repositorien in OpenDOAR

7. re3data.org als Nachweisinstrument für österreichische Repositorien für Forschungsdaten

8. Ausblick

\section{Einleitung}

Der Markt für wissenschaftliche Informationsanbieter hat in den letzten Jahrzehnten einen enormen Konzentrationsprozess durchlaufen, und zwar nicht nur im Bereich der Publikationen, sondern auch hinsichtlich der Produkte für den gesamten akademischen Workflow. Deren Palette reicht von Tools zur Erhebung, Analyse und Archivierung von wissenschaftlichen Veröffentlichungen, Forschungsdaten und Laborbüchern bis hin zu Disseminations- und Evaluationstools. Diese Entwicklung könnte zu einem ,[...] end-to-end lock-in for researchers across every part of their research lifecycle" führen. ${ }^{2}$ Angemerkt sei an dieser Stelle, dass sich dieser Prozess bereits ohne Open Access vollzogen hat, dadurch allerdings auch nicht gebremst wird. ${ }^{3}$ Diese Konzentration ist aus mehreren Gründen problematisch: erstens kontrollieren einige wenige kommerzielle Unternehmen den größtenteils 
mit öffentlichen Mitteln erzeugten Output der Wissenschaft (Platformization), zweitens muss auch weiterhin mit erheblichen Preissteigerungen gerechnet werden und drittens wird es unter diesen Voraussetzungen zukünftig verstärkt zu einem immanenten Bias kommen, weil Produkte eines Anbieters durch andere Produkte desselben Anbieters evaluiert werden, wie z.B. im Falle von Elsevier und SpringerNature.

Um dieser Entwicklung etwas entgegenzusetzen, ist es nicht nur notwendig, alternative, nicht-kommerzielle und von der Wissenschaft kontrollierte Open Science-Infrastrukturen \& -Services (OSIS) langfristig zu fördern, ${ }^{4}$ sondern auch unabdingbar, diese kontinuierlich weiterzuentwickeln, um den ständig steigenden Bedürfnissen von Wissenschaftlerlnnen und Forschungsförderungsorganisationen gerecht werden zu können. ${ }^{5} \mathrm{Zu}$ den OSIS zählt selbstverständlich auch ein Großteil der mittlerweile sehr vielfältigen Landschaft an Repositorien, die bei der Etablierung eines offenen wissenschaftlichen Kommunikationssystems als zentrale Infrastrukturen dienen sollten. ${ }^{6}$

Wenn sie dies nicht ohnehin bereits tun, werden auch österreichische Forschungseinrichtungen in naher Zukunft dazu aufgefordert sein, sich an der GO FAIR Initiative ${ }^{7}$ zu beteiligen und die von ihnen betriebenen elektronischen Infrastrukturen - allen voran die Repositorien - zu einem integralen Bestandteil der angestrebten European Open Science Cloud (EOSC) ${ }^{8}$ werden zu lassen. In einer aktuellen Stellungnahme hat die deutsche Allianz der Wissenschaftsorganisationen ihre Unterstützung für eine europaweite Infrastruktur für datenintensive Forschung durch die EOCS bekundet. ${ }^{9}$ Die Bedeutung von Repositorien für die österreichischen Universitäten im digitalen Zeitalter wurde auch vom österreichischen Bundesminister Heinz Faßmann in einem „Vortrag an den Ministerrat" betont. ${ }^{10}$ Ein geeigneter Zeitpunkt also, um die Entwicklung und den Status Quo von Repositorien in Österreich näher zu betrachten.

\section{Schwierige Anfangsphase für Repositorien in Österreich}

Die deutsche Hochschulrektorenkonferenz hat bereits 2002 - ein Jahr vor der Berliner Erklärung über offenen Zugang zu wissenschaftlichem Wissen ${ }^{11}$ - eine Empfehlung für den Auf- und Ausbau von Hochschulservern für die elektronische Publikation von an der Hochschule entstandenen wissenschaftlichen Arbeiten als infrastrukturelle Maßnahme ausgesprochen. ${ }^{12}$ 2010 hat auch die Österreichische Universitätenkonferenz (uniko) ihren Mitgliedern empfohlen, Repositorien aufzubauen. ${ }^{13}$ Zeitgleich wurde allerdings eine 2009 beschlossene Novelle zum Universitätsgesetz durch das Budgetbegleitgesetz 2010 wieder außer Kraft gesetzt. ${ }^{14}$ Die Novelle hät- 
te das Thema Repositorium auf nationaler Ebene stark forciert, weil sie vorsah, „zur Dokumentation der wissenschaftlichen Leistungen an österreichischen Universitäten [...] eine zentrale Datenbank für wissenschaftliche Veröffentlichungen von Angehörigen der Universitäten (digitales Repositorium) einzurichten" ${ }^{15}$

2012/2013 wurde im Auftrag des Forums Universitätsbibliotheken Österreichs (ubifo) eine Bestandsaufnahme der Open Access-Aktivitäten an den 21 österreichischen öffentlichen Universitäten durchgeführt. Unter anderem erfolgte auch eine Erhebung zum Status des „Grünen Weges zu Open Access“. Während 14 der 21 Universitätsbibliotheken zum Abfragezeitpunkt elektronische Hochschulschriften frei zugänglich im Internet angeboten haben, wurde nur an zwei Universitäten ein Hochschulschriftenserver bzw. ein institutionelles Repositorium im eigentlichen Sinn betrieben, der auch die Archivierung von Pre- und Postprints von wissenschaftlichen Zeitschriftenaufsätzen ermöglichte.

Das Repositorium der Wirtschaftsuniversität Wien (ePubWU) enthielt zum Erhebungszeitpunkt mehr als 1.300 Volltexte (Stand: 28.03.2013). Das neben dem Hochschulschriftenserver (E-THESIS), der 17.700 Volltexte umfasste (Stand: 27.03.2013), neu entwickelte Repositorium der Universität Wien (u:scholar) enthielt zum selben Zeitpunkt über 2.400 Volltexte (Stand: 28.03.2013). ${ }^{16}$

2013 waren von den in OpenDOAR verzeichneten circa 2.250 Repositorien elf Österreich zuzuordnen $(0,48 \%)$, von denen wiederum nur zwei auf österreichische Universitäten entfielen (0,08\%). ${ }^{17}$ Zum Teil handelt es sich bei diesen Repositorien um sehr spezialisierte Repositorien mit geringem Umfang.

In dem 2014 durchgeführten „Census on Open Access Repositories in Germany, Austria and Switzerland“ (Census 2014) wurden nur fünf österreichische Repositorien erfasst. Neben vier universitären Repositorien, die allerdings nur zwei Hochschulen zuzuordnen waren, fand auch das Repositorium der Österreichischen Akademie der Wissenschaften Aufnahme darin. Neben dem Repositorium der Wirtschaftsuniversität Wien scheint die Universität Wien dreimal im Census 2014 auf (Institut für Philosophie, u:scholar, Hochschulschriften-Service).

Insgesamt umfassten die fünf Repositorien zu diesem Zeitpunkt 69.612 Dokumente. Drei der fünf österreichischen Open Access-Repositorien wurden in die Kategorie „mittelgroß“ (1.001 bis 5.000 Dokumente), zwei in die Kategorie „groß“ (ab 5.001 Dokumenten) eingestuft.

Im Census 2014 wurde auch festgehalten, dass keines der österreichischen Open Access-Repositorien den hohen Anforderungen von DINI entsprochen hat. 
Im Open Access Repository Ranking 2015, einer Weiterentwicklung und Aktualisierung der Daten des Census 2014, scheinen mit der Universität Graz (uni=pub) und der Universität Innsbruck (Digitale Bibliothek) zwei weitere österreichische Repositorien auf. ${ }^{18}$

\section{Aufbau von Repositorien im Rahmen von e-Infrastructures Austria}

Zur nachhaltigen Verbesserung dieser Situation wurde in einem der drei Teilprojekte von e-Infrastructures Austria, eines vom Bundesministerium für Wissenschaft, Forschung und Wirtschaft für die Jahre 2014 bis 2016 geförderten Hochschulraumstrukturmittelprojekts, an dem sich 20 öffentliche Universitäten und fünf weitere außeruniversitäre Forschungseinrichtungen beteiligt haben, der Aufbau von Dokumentenservern an jeder einzelnen Partnerinstitution betrieben. ${ }^{19}$ Während Anschaffung und Finanzierung der institutionellen Repositorien als In-kind-Leistung der Projektpartner zu leisten waren, wurden im Rahmen des Kooperationsprojektes unterstützende Dokumente, wie etwa eine Checkliste mit Anforderungen für Dokumente, die in Repositorien eingestellt werden sollen, ${ }^{20}$ eine tabellarische Zusammenstellung und Analyse der an den österreichischen Universitäten eingesetzten Software für Repositorien und deren Besonderheiten ${ }^{21}$ sowie eine Stellenbeschreibung für Repository ManagerInnen ${ }^{22}$ erstellt, die allen Kooperationspartnern zur Verfügung gestellt wurden.

Waren zum Start des Projektes nur an drei Einrichtungen - neben der Universität Wien und der Wirtschaftsuniversität Wien ist hier das IST Austria in Klosterneuburg anzuführen - Repositorien eingerichtet, sollten möglichst alle Kooperationspartner zum Projektabschluss über solche verfügen bzw. deren Aufbau initiiert oder zumindest geplant haben. ${ }^{23}$ Ende 2015 waren bereits an 13 Einrichtungen Repositorien in Betrieb, und an vier weiteren befand sich deren Entwicklung in der Umsetzungsphase. ${ }^{24}$ Bis Ende 2016 erhöhte sich die Zahl der aktiven Repositorien auf 17, vier Einrichtungen befanden sich in der Entwicklungsphase (in der Erhebung „Dokumentenserver 2016“ wurden in dieser Kategorie noch fünf Einrichtungen genannt ${ }^{25}$ ), drei in der Planungsphase. ${ }^{26}$

Der Aufschwung der Repositorien lässt sich auch in einer Analyse der von neun öffentlichen Universitäten in Österreich verabschiedeten Open Access Policies (Stand: 21.12.2016) ablesen. Gab es zu Beginn des Hochschulraumstrukturmittelprojektes e-Infrastructures Austria nur an einer öffentlichen Universität in Österreich eine Open Access Policy (Universität Graz 2013), so erhöhte sich deren Zahl bis zum Ende der Projektlaufzeit 
auf neun (Universität Salzburg 2014, Universität Wien 2014, Universität Klagenfurt 2014, Universität der Technischen Universität Graz 2015, Akademie der bildenden Künste Wien 2015, Universität für Bodenkultur Wien 2015, Wirtschaftsuniversität Wien 2016, Veterinärmedizinische Universität Wien 2016). In sämtlichen Open Access Policies der oben angeführten neun Universitäten wird zur Zweitveröffentlichung ermutigt, diese ausdrücklich empfohlen oder von den Mitarbeiterinnen und Mitarbeitern der eigenen Universität sogar erwartet. ${ }^{27}$

\section{OpenDOAR als Nachweisinstrument für Repositorien}

Das Directory of Open Access Repositories (http://www.opendoar.org) ist eine vom Centre of Research Communications an der University of Nottingham im Jahre 2006 gegründete und laufend aktualisierte Zusammenstellung von Repositorien weltweit, die kostenlos genutzt werden kann. Finanziert wird OpenDOAR von JISC.

Die Registrierung in OpenDOAR kann von jeder Institution, die ein Repositorium betreibt, oder aber auch von Dritten beantragt werden, wobei die Entscheidung über die Aufnahme von einer Redaktion nach Überprüfung diverser Qualitätsstandards getroffen wird. OpenDOAR umfasst derzeit 3.724 Repositorien (Stand: 01.05.2018).

OpenDOAR ermöglicht eine Repositorienrecherche mittels Searchoder Browse-Funktion nach verschiedenen Filtern (Fachgebiet, Land, Dokumententyp, Sprache, Software), stellt aber auch Listen von Repositorien, geordnet nach Ländern und Organisationen zur Verfügung. Darüber hinaus bietet OpenDOAR detaillierte Statistiken.

Aktuell stellt OpenDAOR die umfassendste Datenbank zu Repositorien dar und ermöglicht einen profunden Vergleich hinsichtlich deren Status und Entwicklung weltweit, aber auch auf nationaler Ebene. Der Fokus des vorliegenden Beitrages liegt auf Repositorien, die in OpenDOAR erfasst sind, weil für die Registrierung der betreffenden Repositorien die Einhaltung wichtiger Standards Voraussetzung ist.

\section{5. Österreichische Repositorien in OpenDOAR}

Die folgenden Auswertungen basieren ausschließlich auf einer Recherche nach „Repository by Country“ in OpenDOAR zu „Austria“; dieses weist 30 österreichische Repositorien aus (Stand: 01.05.2018). 


\begin{tabular}{|c|c|c|}
\hline \multicolumn{3}{|c|}{ Österreichische Repositorien in OpenDOAR (Stand: 30.04.2018) } \\
\hline RepositoriumsbetreiberInnen & Repositorium & Dokumente \\
\hline Arbeitskreis Architekturinformatik & $\begin{array}{l}\text { Architektur-Informatik } \\
\text { http://architektur-informatik.scix.net/ } \\
\text { cgi-bin/works/Home }\end{array}$ & 113 \\
\hline $\begin{array}{l}\text { CORP (Competence Center of } \\
\text { Urban and Regional Planning) }\end{array}$ & $\begin{array}{l}\text { REAL CORP } \\
\text { http://repository.corp.at/ }\end{array}$ & 475 \\
\hline CUMINCAD & $\begin{array}{l}\text { CumlnCAD Digital Archive } \\
\text { http://cumincad.architexturez.net/ }\end{array}$ & 12.138 \\
\hline $\begin{array}{l}\text { European Communities Studies } \\
\text { Association Austria (ECSA Austria) }\end{array}$ & $\begin{array}{l}\text { European Research Papers Archive } \\
\text { (ERPA) } \\
\text { http://www2.wu-wien.ac.at/ecsa/ }\end{array}$ & 1.820 \\
\hline FH Burgenland & $\begin{array}{l}\text { People@FH Burgenland } \\
\text { https://people.fh-burgenland.at/ }\end{array}$ & 1.008 \\
\hline $\begin{array}{l}\text { Fonds zur Förderung der wissenschaftli- } \\
\text { chen Forschung (Austrian Science Fund) } \\
\text { (FWF) }\end{array}$ & $\begin{array}{l}\text { FWF-E-Book-Library } \\
\text { https://e-book.fwf.ac.at/ }\end{array}$ & 437 \\
\hline Institut für Höhere Studien, Wien & $\begin{array}{l}\text { IRIHS - Institutional Repository at IHS } \\
\text { http://irihs.ihs.ac.at/ }\end{array}$ & 4.223 \\
\hline $\begin{array}{l}\text { Institute of Science and Technology } \\
\text { Austria (IST Austria) }\end{array}$ & $\begin{array}{l}\text { IST PubRep } \\
\text { https://repository.ist.ac.at/ }\end{array}$ & 700 \\
\hline $\begin{array}{l}\text { International Institute for Applied } \\
\text { Systems Analysis (IIASA) }\end{array}$ & $\begin{array}{l}\text { IIASA PURE } \\
\text { http://pure.iiasa.ac.at/ }\end{array}$ & 14.582 \\
\hline Johannes Kepler Universität Linz & $\begin{array}{l}\text { JKU ePub } \\
\text { http://epub.jku.at/ }\end{array}$ & 2.051 \\
\hline Karl-Franzens-Universität Graz & $\begin{array}{l}\text { uni }=p u b \text { (unipub) } \\
\text { http://unipub.uni-graz.at/ }\end{array}$ & 16.480 \\
\hline $\begin{array}{l}\text { Karl-Franzens-Universität Graz, Zentrum } \\
\text { für Informationsmodellierung - } \\
\text { Austrian Centre for Digital Humanities }\end{array}$ & $\begin{array}{l}\text { GAMS - Asset Management System for } \\
\text { the Humanities } \\
\text { http://gams.uni-graz.at/ }\end{array}$ & 37.554 \\
\hline $\begin{array}{l}\text { Ludwig Boltzmann Institut für Health } \\
\text { Technology Assessment (LBI-HTA) }\end{array}$ & $\begin{array}{l}\text { Dokumentenserver des LBI-HTA } \\
\text { http://eprints.hta.lbg.ac.at/ }\end{array}$ & 880 \\
\hline Medizinische Universität Wien & $\begin{array}{l}\text { MedUni Wien ePub } \\
\text { http://repositorium.meduniwien.ac.at/ }\end{array}$ & 1.329 \\
\hline Oberösterreichische Landesbibliothek & $\begin{array}{l}\text { Digitale Landesbibliothek } \\
\text { Oberösterreich } \\
\text { http://digi.landesbibliothek.at/ }\end{array}$ & 5.177 \\
\hline $\begin{array}{l}\text { Österreichische Akademie der } \\
\text { Wissenschaften }\end{array}$ & $\begin{array}{l}\text { Elektronisches Publikationsportal der } \\
\text { Österreichischen Akademie der } \\
\text { Wissenschaften (epub.oeaw) } \\
\text { http://epub.oeaw.ac.at/ }\end{array}$ & 42.463 \\
\hline Paris Lodron Universität Salzburg & $\begin{array}{l}\text { ePLUS } \\
\text { http://eplus.uni-salzburg.at/ }\end{array}$ & 1.042 \\
\hline
\end{tabular}




\begin{tabular}{|c|c|c|}
\hline SG3 Knowledge Network OG & $\begin{array}{l}\text { Fluorophores.org } \\
\text { http://www.fluorophores.tugraz.at/ }\end{array}$ & 910 \\
\hline SG3 Knowledge Network OG & $\begin{array}{l}\text { PiezoMat.org } \\
\text { http://www.PiezoMat.org/ }\end{array}$ & 315 \\
\hline Technische Universität Graz & $\begin{array}{l}\text { TUGraz OPEN Library } \\
\text { http://openlib.tugraz.at/ }\end{array}$ & 346 \\
\hline Technische Universität Wien & $\begin{array}{l}\text { Publication Database of the Vienna } \\
\text { University of Technology } \\
\text { http://publik.tuwien.ac.at/start. } \\
\text { php?lang=2 }\end{array}$ & $\begin{array}{l}\text { Keine } \\
\text { Angabe }\end{array}$ \\
\hline Technische Universität Wien & $\begin{array}{l}\text { reposiTUm } \\
\text { http://repositum.tuwien.ac.at/ }\end{array}$ & 11.455 \\
\hline $\begin{array}{l}\text { textfeld society for advancement of } \\
\text { academic potential }\end{array}$ & $\begin{array}{l}\text { textfeld } \\
\text { http://www.textfeld.ac.at/ }\end{array}$ & 754 \\
\hline Universität für Bodenkultur Wien & $\begin{array}{l}\text { BOKU:epub (Publikationsserver der } \\
\text { Universitätsbibliothek Bodenkultur Wien) } \\
\text { http://epub.boku.ac.at/ }\end{array}$ & 2.768 \\
\hline Universität Innsbruck & $\begin{array}{l}\text { University of Innsbruck Digital Library } \\
\text { http://diglib.uibk.ac.at/ }\end{array}$ & 5.454 \\
\hline Universität Wien & $\begin{array}{l}\text { Elektronisch archivierte Theorie - } \\
\text { Sammelpunkt } \\
\text { http://sammelpunkt.philo.at:8080/ }\end{array}$ & 1.877 \\
\hline Universität Wien & $\begin{array}{l}\text { Phaidra (Permanent Hosting, Archiving } \\
\text { and Indexing of Digital Resources and } \\
\text { Assets) } \\
\text { https://phaidra.univie.ac.at/ }\end{array}$ & 54.229 \\
\hline Universität Wien & $\begin{array}{l}\text { u:scholar } \\
\text { http://uscholar.univie.ac.at/ }\end{array}$ & 3.870 \\
\hline $\begin{array}{l}\text { Verband kirchlich-wissenschaftlicher } \\
\text { Bibliotheken (VkwBB) }\end{array}$ & $\begin{array}{l}\text { KiDokS (Kirchlicher DokumentenServer } \\
\text { der AKThB und des VkwB) } \\
\text { http://opus.bsz-bw.de/kidoks/ }\end{array}$ & 740 \\
\hline Wirtschaftsuniversität Wien (WU) & $\begin{array}{l}\text { ePubWU (ePubWU Institutional } \\
\text { Repository) } \\
\text { http://epub.wu.ac.at/ }\end{array}$ & 2.789 \\
\hline \multicolumn{3}{|c|}{$\begin{array}{l}\text { Bei den in der Spalte „Dokumente" angegebenen Werten handelt es sich zu einem überwiegenden } \\
\text { Teil um die Anzahl an Dokumenten, die das jeweilige Repositorium zum Zeitpunkt der Ersterfassung } \\
\text { durch OpenDOAR beinhaltet hat. Zwei Stichproben ergaben, dass aktuell bereits deutlich mehr } \\
\text { Objekte in die jeweiligen Repositorien hochgeladen wurden. }\end{array}$} \\
\hline
\end{tabular}

Tab. 1: Österreichische Repositorien in OpenDOAR (Stand: 30.04.2018) 
Startete OpenDOAR 2006 mit zwei Repositorien aus Österreich, so erhöhte sich deren Anzahl bis zum Jahresende 2006 auf fünf. 2007 waren sieben, 2008 acht Repositorien gelistet. 2009 gab es keine Veränderung, erst 2010 kam ein weiteres hinzu, sodass OpenDOAR zu diesem Zeitpunkt insgesamt neun österreichische Repositorien verzeichnete. Veränderte sich im Jahre 2011 zwar nichts, so stieg deren Zahl 2012 auf elf und 2013, dem Jahr vor Beginn des Hochschulraumstrukturmittelprojektes e-Infrastructures Austria, auf 13 an.

2014 erhöhte sich die Zahl der österreichischen Repositorien auf 17, 2015 auf 22 und 2016 auf 28. Somit hat sich die Anzahl während der dreijährigen Projektphase von e-Infrastructures (2014-2016) mehr als verdoppelt.

2017 und 2018 wurde je ein Repositorium aus Österreich in OpenDOAR registriert, sodass die Gesamtzahl derzeit 30 lautet (Stand: 01.05.2018).

Waren 2013 von etwa 2.250 in OpenDOAR erfassten Repositorien elf Österreich zuzuordnen (0,48\%), so beträgt der Anteil 201830 von 3.724 Repositorien (0,81\%).

Waren 2014 nur zwei der österreichischen Repositorien in OpenDOAR Universitäten zuzuweisen (0,08\%), so erhöhte sich deren Anzahl 2018 auf zwölf $(0,32 \%)$.

Betrieben werden die 30 Repositorien von 28 Institutionen. Sowohl SG3 Knowledge Network OG als auch die Universität Wien betreiben jeweils zwei Repositorien.

\section{Spezifika der österreichischen Repositorien in OpenDOAR}

\section{Dokumententypen und Fachgebiete}

Analysiert man die 30 in OpenDOAR registrierten österreichischen Repositorien nach den jeweils erfassten Dokumententypen, so zeigt sich ein sehr heterogenes Bild. Fünf Repositorien (16\%) bieten keine Informationen zu den aufgenommenen Dokumententypen an. Zwölf Repositorien (40\%) beinhalten Publikationen (Artikel, Bücher), sieben (23\%) Konferenzbeiträge, zehn Dissertationen (33\%), neun (30\%) nichtpubliziertes Material und elf (36\%) andere Materialien (Datasets, Multimedia, Lernobjekte, Patente).

Hinsichtlich ihrer fachlichen Ausrichtung decken die österreichischen in OpenDOAR verzeichneten Repositorien eine große Bandbreite an wissenschaftlichen Fachgebieten ab. So zählen 14 Repositorien (47\%) zur Gruppe der interdisziplinären Repositorien. Fünf (17\%) haben einen thematischen Schwerpunkt auf Business and Economics, und je vier (je 13\%) werden mit 
den fachlichen Ausrichtungen Science General, Health and Medicine, Computers and IT, Geography and Regional Studies, Philosophy and Religion sowie Law and Information Science betrieben.

Für alle anderen Fachgebiete weist die Statistik nur zwischen ein und zwei österreichische Repositorien in OpenDOAR aus.

\section{Umfang}

In 29 der 30 erfassten österreichischen Repositorien sind laut OpenDOAR insgesamt 228.044 elektronische Dokumente erfasst; für die Publication Database of the Vienna University of Technology ist keine Größenordnung angeführt.

In die Kategorie der kleineren Repositorien (bis zu 1.000 Dokumente) fallen zehn, in jene der mittleren (von 1.000 bis 9.900 Dokumente) zwölf und in jene der großen (über 10.000 Dokumente) sieben Repositorien.

Die kleineren Repositorien umfassen insgesamt 5.675 Dokumente, sodass jedes der zehn Repositorien in dieser Gruppe im Durchschnitt 567 Dokumente beinhaltet.

Die Gesamtzahl der Dokumente in der Kategorie der mittleren Repositorien beträgt 33.427; jedes der zwölf Repositorien dieser Gruppe enthält somit im Durchschnitt 2.785 Dokumente.

Die als große kategorisierten Repositorien umfassen insgesamt 188.942 Dokumente; somit beträgt die Anzahl an Dokumenten in jedem der sieben Repositorien in dieser Gruppe durchschnittlich 26.991.

\section{Software und OAI-Schnittstelle}

Für sechs der 30 in OpenDOAR erfassten österreichischen Repositorien werden hinsichtlich der verwendeten Software keine näheren Angaben gemacht (20\%). Am verbreitetsten sind demnach die Softwarelösungen EPrints und Visual Library, die für jeweils sieben Repositorien eingesetzt werden (je $23 \%$ ), gefolgt von Fedora, das viermal zum Einsatz kommt (13\%). Je einmal genutzt werden DSpace, Hyperwave, Opus und SciX sowie je eine Eigenentwicklung basierend auf HTML bzw. PHP \& MySQL (je $3 \%$ ).

25 der 30 in OpenDOAR verzeichneten Repositorien verfügen über eine OAI-Schnittstelle (83\%), für fünf ist eine solche nicht angeführt (17\%).

\section{Policies}

Im Bereich der Policies weisen die österreichischen Repositorien in OpenDOAR noch Defizite auf. 
Sechs Repositorien (20\%) gestatten eine Nutzung der Metadaten für nichtkommerzielle Zwecke (Elektronisch archivierte Theorie - Sammelpunkt, Elektronisches Publikationsportal der Österreichischen Akademie der Wissenschaften, ePubWU, European Research Papers Archive, IIASA PURE, REAL CORP); für den Großteil der Repositorien gibt es diesbezüglich keine Policy.

Ein ähnliches Bild zeigt sich bei der Frage nach der Gestattung der Nachnutzung der elektronischen Volltexte: diese wird nur für die Inhalte von fünf Repositorien (17\%) explizit eingeräumt.

Auch Content Policy bzw. Submission Policy sind weitgehend undefiniert bzw. unbekannt; nur sechs Repositorien (20\%) verfügen in diesem Zusammenhang über eine diesbezügliche Leitlinie.

Nur drei der 30 österreichischen Repositorien (10\%) verfügen über eine Policy zum Thema Langzeitarchivierung (Elektronisches Publikationsportal der Österreichischen Akademie der Wissenschaften, ePubWU, IIASA PURE).

\section{7. re3data.org als Nachweisinstrument für österreichische Repositorien für Forschungsdaten}

Vergleicht man die in OpenDAOR erfassten österreichischen Repositorien mit den in re3data.org: Registry of Research Data Repositories (https:// www.re3data.org/) aufgenommenen 32 Repositorien für Forschungsdaten, die in Österreich bzw. in Kooperation mit in Österreich ansässigen Einrichtungen betrieben werden (Browse by country: „Austria“), so ergibt sich folgendes Bild: 16 tragen länderspezifische Top-Level-Domains (AT 12, SE 1, NO 1, UK 1, EU 1), 16 generische Top-Level-Domains (ORG 12, GOV 2, EDU 1, INT 1).

Die zwölf Repositorien für Forschungsdaten mit der länderspezifischen Top-Level-Domain AT sind folgenden Einrichtungen zuzuordnen:

- Core Facility AUSSDA / Vienna University, Library (AUSSDA: The Austrian Social Science Data Archive; http://www.aussda.at)

- CLARIAH-AT / Austrian Center for Digital Humanities / CLARIN ERIC / Österreichische Akademie der Wissenschaften (ARCHE: A Resource Centre for the HumanitiEs; https://arche.acdh.oeaw. ac.at/browser/)

- Medical University of Innsbruck, Biocenter, Division of Bioinformatics / APERIM / European Commission, Horizon 2020 (The Cancer Immunome Atlas: TCIA; https://tcia.at/home) 
- GGOS / Technische Universität Wien, Department of Geodesy and Geoinformation / FWF Der Wissenschaftsfonds (GGOS Atmosphere; http://ggosatm.hg.tuwien.ac.at/proj_ggosatm.html)

- University of Music and Performing Arts Vienna (mdw Repository; https://repo.mdw.ac.at)

- Karl-Franzens-Universität Graz, Zentrum für Informationsmodellierung (GAMS: Geisteswissenschaftliches Asset Management System; http://gams.uni-graz.at/context:gams)

- Universität Wien / Phaidra Network (Phaidra Universität Wien: Permanent Hosting, Archiving and Indexing of Digital Resources and Assets; https://phaidra.univie.ac.at/)

- Universität Wien / Vorarlberger Landesbibliothek (volare: Vorarlberger Landesrepositorium; https://www.vorarlberg.at/volare)

- CLARIN-ERIC / Austrian Academy of Sciences, Austrian Centre for Digital Humanities / Österreich, Bundesministerium für Wissenschaft, Forschung und Wirtschaft / Österreichische Akademie der Wissenschaften (CLARIN Centre Vienna: CCV; https://clarin.oeaw.ac.at/ccv/)

- Virtual Atomic and Molecular Data Centre consortium / University of Innsbruck, Institute for Ion Physics and Applied Physics (IDEADB: Innsbruck Dissociative Electron Attachment Database; http://ideadb.uibk.ac.at)

- Zentralanstalt für Meteorologie und Geodynamik / Climate Change Centre Austria (CCCA Data Centre: Climate Change Centre Austria Data Centre; https://data.ccca.ac.at/)

- Institute of Science and Technology Austria (IST DataRep: Institute of Science and Technology DataRep; https://datarep.app.ist.ac.at/)

Drei der 12 Repositorien mit der länderspezifischen Top-Level-Domain sind auch in OpenDOAR erfasst (GAMS, Phaidra, IST DataRep).

Von den 12 Repositorien mit der Top-Level-Domain ORG werden acht von der IAEA (International Atomic Energy Agency) betrieben.

An drei Repositorien (ARCHE, CLARIN Centre Vienna, GAMS) wurde bereits das Data Seal of Approval (DSA, https://www.datasealofapproval. org/), an eines (ARCHE) das CoreTrustSeal (CTS, https://www.coretrustseal.org/ $)^{28}$ vergeben.

\section{Ausblick}

Nachdem Österreich in den Jahren seit der „Berliner Erklärung über offenen Zugang zu wissenschaftlichem Wissen“ (2003) bei Entwicklung und 
Betrieb von Repositorien lange Zeit hinter der internationalen Entwicklung zurückgeblieben ist, erfolgte im Rahmen des Hochschulraumstrukturmittelprojektes e-Infrastructures Austria ein bemerkenswerter Aufholprozess, insbesondere an den öffentlichen Universitäten. Dies spiegelt sich auch in der Zahl der in OpenDOAR erfassten österreichischen Repositorien wider und ist im Hinblick auf die zentrale Bedeutung von OpenDOAR ein wesentlicher Entwicklungsschritt für die einzelnen institutionellen Repositorien, die zum Teil nur über eine relativ kleine Zahl an Dokumenten verfügen, deren Sichtbarkeit allerdings in der Zusammenschau der Repositorien auf globaler Ebene deutlich erhöht wird. In diesem Zusammenhang ist es aus nationaler Sicht bedauerlich, dass (noch immer) nicht alle österreichischen Repositorien in OpenDOAR verzeichnet sind. Gerade im bibliothekarischen Umfeld ist der Wert diverser Directories unbestritten. Dies sollte auch eine Ermunterung sein, die Daten des eigenen Repositoriums an die Redaktion von OpenDOAR zu übermitteln bzw. gegebenenfalls Korrekturen zu veranlassen.

Seit Mai 2018 steht OpenDOAR auch in einer überarbeiten Version, derzeit noch als Beta-Version, zur Verfügung; diese bietet allerdings (noch) nicht alle bisherigen Suchoptionen (http://v2.opendoar.sherpa.ac.uk/api.html).

Ein Desiderat stellt derzeit noch die Erfassung der österreichischen Repositorien in der DINI Liste der Repositorien dar, in der derzeit 235 Repositorien ausgewiesen sind (https://dini.de/dini-zertifikat/liste-der-repositorien/). Die DINI Liste verfolgt den Anspruch, Repositorien in Deutschland, Österreich und der Schweiz zu verzeichnen und gegebenenfalls auch darzustellen, ob ein DINI Zertifikat erworben wurde. Österreich ist aktuell in der DINI Liste mit MedUni Wien ePub, dem von der Universitätsbibliothek der Medizinischen Universität Wien betriebenen Repositorium, und KiDokS, dem Kirchlichen DokumentenServer der Arbeitsgemeinschaft der katholisch-theologischen Bibliotheken (AKThB) und des Verbandes kirchlich wissenschaftlicher Bibliotheken (VkwB) mit dem Standort Linz, nur zweimal vertreten. KiDoks ist ein Dokumentenserver für 14 Einrichtungen in Deutschland, Österreich und der Schweiz.

Abschließend kann für die Situation in Österreich festgehalten werden, dass der Anfang - der Aufbau und Betrieb von Repositorien - nun vielerorts gemacht worden ist. In unmittelbarer Zukunft sind aber noch viele weitere Hürden, wie z.B. Weiterentwicklung, Koordination, Kollaboration und Synergiebildung, Vernetzung und „Einbettung“ in die European Open Science Cloud, Bewusstseinsbildung sowie Aus- und Weiterbildung, zu bewältigen, um die Etablierung eines offenen wissenschaftlichen Kommunikationssystems zu unterstützen. ${ }^{29} 30$ 
Mag. Bruno Bauer

ORCID: http://orcid.org/0000-0002-4729-331X Medizinische Universität Wien, Universitätsbibliothek

E-Mail: bruno.bauer@meduniwien.ac.at

Mag. Andreas Ferus, MSc ORCID: http://orcid.org/0000-0003-2509-0009 Akademie der bildenden Künste Wien, Universitätsbibliothek E-Mail: a.ferus@akbild.ac.at

1 Schonfeld, Roger C. (2018): Workflow Lock-in - A Taxonomy. Online: https://scholarlykitchen.sspnet.org/2018/01/02/workflow-lock-taxonomy/ (Zugriff: 12.06.2018).

2 Posada, Alejandro; Chen, George (2017): Preliminary Findings - Rent Seeking by Elsevier. Publishers are increasingly in control of scholarly infrastructure and why we should care - A Case Study of Elsevier. Online: http://knowledgegap.org/index.php/sub-projects/rent-seekingand-financialization-of-the-academic-publishing-industry/preliminaryfindings/ (Zugriff: 12.06.2018).

3 Schonfeld, Roger C. (2018): Big Deal - Should Universities Outsource More Core Research Infrastructure? New York: Ithaka S+R. https://doi. org/10.18665/sr.306032

4 Global Sustainability Coalition for Open Science Services (SCOSS). Online: http://scoss.org/

5 Lovett, Julia A.; Rathemacher, Andrée J.; Boukari, Divana; Lang, Corey (2017): Institutional Repositories and Academic Social Networks: Competition or Complement? A Study of Open Access Policy Compliance vs. ResearchGate Participation. In: Journal of Librarianship and Scholarly Communication 5(1), eP2183. http://doi.org/10.7710/21623309.2183; Wipperman, Sarah; Martin, Shawn; Bowley, Chealsye (2018): Balancing influence in a shifting scholarly communication landscape. Creating library-owned, community-aligned infrastructure through individual, local, and community action. In: College \& Research Libraries News 79(5), 244. https://doi.org/10.5860/crln.79.5.244

6 Behaviours and Technical Recommendations of the COAR Next Generation Repositories Working Group (28.11.2017). Online: https://www. coar-repositories.org/files/NGR-Final-Formatted-Report-cc.pdf

7 GO FAIR Initiative. Online: https://www.go-fair.org/

8 European Open Science Cloud. Online: https://ec.europa.eu/research/ 
openscience/index.cfm?pg=open-science-cloud (Zugriff: 12.06.2018).

9 Allianz der Wissenschaftsorganisationen: Stellungnahme der Allianz der Wissenschaftsorganisationen zu einer European Open Science Cloud (EOSC), 20.03.2018. Online: https://www.fraunhofer.de/content/ dam/zv/de/ueber-fraunhofer/wissenschaftspolitik/10/20180320_Stellungnahme\%20Allianz\%20zur\%20EOSC.pdf (Zugriff: 12.06.2018).

10 Faßmann, Heinz: Vorhaben des Bundesministers für Bildung, Wissenschaft und Forschung zur ZUKUNFT DIGITALISIERUNG - Die österreichischen Universitäten am Weg ins digitale Zeitalter. Vortrag an den Ministerrat, 07.06.2018. Online: https://www.bundeskanzleramt. gv.at/documents/131008/877075/21_9_mrv.pdf/1e321 eea-066c4d71-8143-b757d21cfe21 (Zugriff: 12.06.2018)

11 Berliner Erklärung über offenen Zugang zu wissenschaftlichem Wissen, 22.10.2003. Online: http://www.cbs.mpg.de/329920/Berliner-Erklaerung-ueber-den-offenen-Zugang-zu-wissenschaftlichem-Wissen.pdf (Zugriff: 12.06.2018).

12 Hochschulrektorenkonferenz: Zur Neuausrichtung des Informationsund Publikationssystems der deutschen Hochschulen. Empfehlung des 198. Plenums vom 5. November 2002. Online: https://www.hrk. de/uploads/tx_szconvention/Empfehlung_Bibliothek.pdf (Zugriff: 12.06.2018)

13 Empfehlungen der Österreichischen Universitätenkonferenz (uniko) zu einer Open Access-Politik der Universitäten. Beschluss vom 12. Jänner 2010. Online: http://www.rritrends.res-agora.eu/uploads/12/UnikoEmpfehlungen_Open_Access_01_2010.pdf(Zugriff: 12.06.2018)

14 Pauser, Josef (2011): Error! - „Zentrale Datenbank für wissenschaftliche und künstlerische Arbeiten" wurde Ende 2010 wieder beerdigt. In: Mitteilungen der Vereinigung Österreichischer Bibliothekarinnen und Bibliothekare 64(1), S. 120-124.

15 Bauer, Bruno; Stieg, Kerstin (2010): Open Access Publishing in Österreich 2010. In: Bibliotheksdienst 44(7), S. 700-710. https://doi. org/10.1515/bd.2010.44.7.700

16 Bauer, Bruno; Gumpenberger, Christian; Haas, Ingrid; Katzmayr, Michael; Ramminger, Eva; Reinitzer, Doris (2013): Open Access Bestandsaufnahme an österreichischen Universitäten: Ergebnisse einer Umfrage im Auftrag des Forums Universitätsbibliotheken Österreichs (UBIFO). In: Mitteilungen der Vereinigung Österreichischer Bibliothekarinnen und Bibliothekare 66(3/4), S. 535-558. Online: http://eprints.rclis. org/20968/ (Zugriff: 12.06.2018)

17 Blechl, Guido; Blumesberger, Susanne (2013): Open Access an öster- 
reichischen Universitätsbibliotheken. In: Bauer, Bruno; Gumpenberger, Christian; Schiller, Robert (Hrsg.): Universitätsbibliotheken im Fokus. Aufgaben und Perspektiven der Universitätsbibliotheken an öffentlichen Universitäten in Österreich. (Schriften der Vereinigung Österreichischer Bibliothekarinnen und Bibliothekare 13). Wolfgang Neugebauer Verlag GesmbH: Graz-Feldkirch, S. 198-215. Online: http://eprints.rclis. org/24292/ (Zugriff: 12.06.2018)

18 Bader, Tabea (2016): Open-Access-Repositorien in der Schweiz und Österreich - Auswertung des 2014 Census on Open Access Repositories. In: LIBREAS. Library Ideas 29. Online: http://libreas.eu/ ausgabe29/13bader/ (Zugriff: 12.06.2018)

19 Flicker, Katharina; Sánchez Solís, Barbara; Budroni, Paolo (Hrsg.) (2017): e-Infrastructures Austria: Projektbericht 2014-2016. Wien: eInfrastructures Austria. URI: https://hdl.handle.net/11353/10.460779

20 Blumesberger, Susanne; Danowski, Patrick; Gründhammer, Veronika; Haas, Ingrid; Novotny, Gertraud; Ohrtmann, Linda; Paril, Cornelia; Torggler, Elisabet (2015): Anforderungen an Repositorys für Dokumente. Wien: e-Infrastructures Austria, 2014-2016. URI: http://hdl. handle.net/10760/25437

21 Birkner, Michael; Ganguly, Raman; Gonter, Gerhard; Ladurner, Christoph; Mitterhuber, David (2016): e-Infrastructures Austria: Cluster D: Technischer Überblick über Repositories Dokumentenserver der eInfrastructures Austria Partnereinrichtungen. Wien: e-Infrastructures Austria, 2014-2016. URI: https://hdl.handle.net/11353/10.459177

22 Blumesberger, Susanne; Danowski, Patrick; Gründhammer, Veronika; Haas, Ingrid; Novotny, Gertraud; Ohrtmann, Linda; Paril, Cornelia; Torggler, Elisabet (2015): Modulare Stellenbeschreibung „Repository Manager" (w/m). Wien: e-Infrastructures Austria, 2014-2016. URI: http://hdl.handle.net/10760/25436

23 Bauer, Bruno; Budroni, Paolo; Ferus, Andreas; Ganguly, Raman; Ramminger, Eva; Sánchez Solís, Barbara (2015): e-Infrastructures Austria 2014: Bericht über das erste Jahr des Hochschulraumstrukturmittelprojekts zur Förderung für den koordinierten Aufbau und die kooperative Weiterentwicklung von Repositorieninfrastrukturen. In: Mitteilungen der Vereinigung Österreichischer Bibliothekarinnen und Bibliothekare 68(1), S. 91-118. https://doi.org/10.31263/voebm.v68i1.1000

24 Bauer, Bruno; Budroni, Paolo; Ferus, Andreas; Ganguly, Raman; Ramminger, Eva; Sánchez Solís, Barbara (2016): e-Infrastructures Austria 2015: Bericht über das zweite Jahr des Hochschulraumstrukturmittelprojekts für den koordinierten Aufbau und die kooperative Weiterent- 
wicklung von Repositorieninfrastrukturen. In: Mitteilungen der Vereinigung Österreichischer Bibliothekarinnen und Bibliothekare 69(1), S. 9-40. https://doi.org/10.31263/voebm.v69i1.1394

25 Bedenig, Christoph; Blumesberger, Susanne; Danowski, Patrick; Ganguly, Raman; Gründhammer, Veronika; Haas, Ingrid; Novotny, Gertraud; Paril, Cornelia; Preza, José Luis; Torggler, Elisabet (2016): e-Infrastructures Austria: Cluster A: Dokumentenserver in den e-Infrastructures Austria Partnereinrichtungen: Erhebung 2016. Wien: e-Infrastructures Austria. Online: https://phaidra.univie.ac.at/view/o:459231

26 Bauer, Bruno; Budroni, Paolo; Ferus, Andreas; Ganguly, Raman; Ramminger, Eva; Sánchez Solís, Barbara (2017): e-Infrastructures Austria 2016: Bericht über das dritte Jahr des Hochschulraumstrukturmittelprojekts für den koordinierten Aufbau und die kooperative Weiterentwicklung von Repositorieninfrastrukturen. In: Mitteilungen der Vereinigung Österreichischer Bibliothekarinnen und Bibliothekare 70(1), S. 66-93. https://doi.org/10.31263/voebm.v70i1.1834

27 Bauer, Bruno; Ferus, Andreas; Schilhan, Lisa (2016): Checkliste „Open Access Policies": Analyse von Open Access Policies öffentlicher Universitäten in Österreich. In: Mitteilungen der Vereinigung Österreichischer Bibliothekarinnen und Bibliothekare 69(3), S. 447-475. https://doi. org/10.31263/voebm.v69i3.1734

28 Dillo, Ingrid; Leeuw, Lisa de (2018): CoreTrustSeal. In: Mitteilungen der Vereinigung Österreichischer Bibliothekarinnen und Bibliothekare 71(1), S. 162-170. http://doi.org/ 10.31263/voebm.v71i1.1981

29 Torggler, Andrea; Andrae, Magdalena (2018): Aus dem Leben einer/s Repman - ein Bericht aus dem österreichischen „Netzwerk für RepositorienmanagerInnen“. In: Mitteilungen der Vereinigung Österreichischer Bibliothekarinnen und Bibliothekare 71(1), S. 107-124. https// doi.org/10.31263/voebm.v71i1.1986

30 Behaviours and Technical Recommendations of the COAR Next Generation Repositories Working Group (28.11.2017). Online: https:// www.coar-repositories.org/files/NGR-Final-Formatted-Report-cc.pdf 